\title{
Simultaneous Osteoperiosteal Autologous Iliac Crest Graft and Lateral Meniscus Allograft Transplantation for Osteochondral Lesion with Bony Defect and Lateral Discoid Meniscus Tear
}

\author{
Dhong Won Lee, $\mathrm{MD}^{1}$, Jin Goo Kim, $\mathrm{MD}, \mathrm{PhD}^{2}$, Jeong $\mathrm{Ku} \mathrm{Ha}, \mathrm{MD}^{3}$, and Woo Jong Kim, $\mathrm{MD}^{4}$ \\ ${ }^{1}$ Department of Orthopedic Surgery, Daejeon Military Hospital, Daejeon; ${ }^{2}$ Department of Orthopedic Surgery, Konkuk University Medical Center, Seoul; ${ }^{3}$ Department \\ of Orthopedic Surgery, Inje University Seoul Paik Hospital, Seoul; ${ }^{4}$ Department of Orthopedic Surgery, Soonchunhyang University Cheonan Hospital, Choenan, Korea
}

\begin{abstract}
The optimal treatment for combined osteochondritis dissecans (OCD) with considerable bony defect of the lateral femoral condyle (LFC) and torn discoid lateral meniscus is unclear. We present a case of a 15-year-old female who was a gymnast and had a large OCD lesion in the LFC combined with deficiency of the lateral meniscus. The patient underwent the "one-step" technique of osteoperiosteal autologous iliac crest graft and lateral meniscus allograft transplantation after a failure of meniscectomy with repair at another hospital. Twenty-four months postoperatively, clinical results were significantly improved. Follow-up imaging tests and second-look arthroscopy showed well incorporated structured bone graft and fibrous cartilage regeneration as well as stabilized lateral meniscus allograft. She could return to her sport without any pain or swelling. This "one-step" surgical technique is worth considering as a joint salvage procedure for massive OCD lesions with torn discoid lateral meniscus.
\end{abstract}

Keywords: Knee, Meniscus, Cartilage, Autograft, Transplantation

Osteochondritis dissecans (OCD) is a pathological condition that appears to destruct subchondral bone with secondary effects on the overlying articular cartilage, which eventually results in osteochondral separation including a loose body ${ }^{1}$. The reported causes of OCD include genetic, traumatic, ischemic, and persistent accessory ossification ${ }^{11}$. Discoid lateral meniscus that could produce repetitive abnormal stress on the lateral femoral condyle (LFC) has been known as one of the factors of $\mathrm{OCD}^{11}$.

There has been no optimal treatment for OCD of the LFC associated with discoid lateral meniscus tears. Furthermore, if an

Received September 13, 2015; Revised October 26, 2015;

Accepted November 3, 2015

Correspondence to: Jin Goo Kim, MD, PhD

Department of Orthopedic Surgery, Konkuk University Medical Center,

120-1 Neungdong-ro, Gwangjin-gu, Seoul 05030, Korea

Tel: +82-2-2030-7606, Fax: +82-2-2270-0023

E-mail: boram107@hanmail.net

This is an Open Access article distributed under the terms of the Creative Commons Attribution Non-Commercial License (http://creativecommons.org/licenses/by-nc/4.0/) which permits unrestricted non-commercial use, distribution, and reproduction in any medium, provided the original work is properly cited.
OCD lesion is massive and requires an adequate reconstruction of the osteochondral defect, the treatment is more challenging. Treatment of OCD alone without any procedure on the torn discoid lateral meniscus would result in persistent symptoms and ultimately lead to a failure of the treatment of OCD and progression of arthritis ${ }^{1)}$. Hence, the strategy for combined massive OCD and torn discoid lateral meniscus should be carefully considered.

According to a recent study, severe OCD lesions of the talus could be successfully treated with bony periosteum-covered iliac crest plug transplantation ${ }^{2}$. The study showed that the periosteum of the iliac crest containing many stem cells have a capacity of differentiating into chondrocytes and fibro-cartilage. In addition, their technique could provide osteochondral stability in both osseous and chondral levels without donor site morbidity. Above all, structured bone grafts are needed to fill the osseous defect and prevent collapse of the reconstruction, and the anterior iliac crest has been known to be effect under compressive and shear stresses $^{2}$. Inspired by the technique, we thought the weight bearing capacity of the structured bone graft from the anterior iliac crest could be applied to the femoral condyle of the knee. 
Here, we present a case of a 15-year-old female who was a gymnast and had a large OCD lesion in the LFC combined with deficiency of the lateral meniscus. The patient was treated with a "one-step" procedure of osteoperiosteal autologous iliac crest graft and lateral meniscus allograft transplantations.

\section{Case Report}

A 15-year-old female slipped down during gymnastics practice and had a right knee pain localizing to the lateral side. She went to a hospital 5 days after the injury and was diagnosed with OCD of the LFC and anterior defect with posterocentral shift of the discoid lateral meniscus. The patient underwent arthroscopic partial meniscectomy with repair of the lateral meniscus and multiple drilling at the LFC 2 weeks after the injury at the hospital. During 3 months after the surgery, however, her pain had worsened and she could not walk well.

Eventually, the patient was transferred to our clinic at 4 months after the first surgery. Physical examination revealed tenderness on the lateral joint line of the right knee and positive McMurray test on internal rotation of the tibia. Plain radiographs of the right knee showed an obvious OCD lesion with a large bony defect in the LFC and narrowing of the lateral joint space (Fig. 1). Standing alignment of the lower legs demonstrated about 2 degrees of slight valgus of the right knee compared to the left knee (Fig. 2). Magnetic resonance imaging (MRI; Intera Achieva, Philips, Amsterdam, The Netherlands) of the right knee revealed a 2.5 $\mathrm{cm} \times 3 \mathrm{~cm}$ sized lesion mainly in the posterolateral area of the LFC (Fig. 3). The lesion progressed more compared with the preoperative initial MRI obtained 5 months ago (Fig. 3). The MRI also demonstrated a complex tear of the lateral meniscus where flaps adhered to the OCD lesion of the LFC (Fig. 3). The patient underwent arthroscopic total meniscectomy of the lateral meniscus and debridement of the OCD lesion first. At that time, since the torn lateral meniscus showed severe plastic deformation, we considered there was no regrowth potential of the remnant lateral meniscus. The OCD lesion treated with multiple drilling at another hospital showed a large osteochondral defect without any regeneration (Fig. 4). Therefore, we planned to perform simultaneous osteoperiosteal autologous iliac crest graft implantation for the massive OCD lesion and lateral meniscus allograft trans-

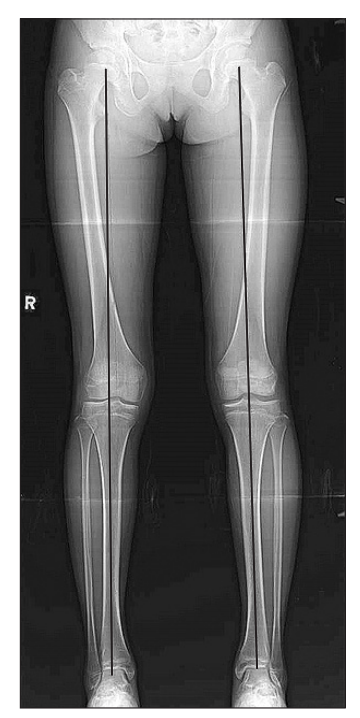

Fig. 2. Standing lower extremity radiograph showing slight valgus of the right knee (about 2 degrees compared to the left knee).
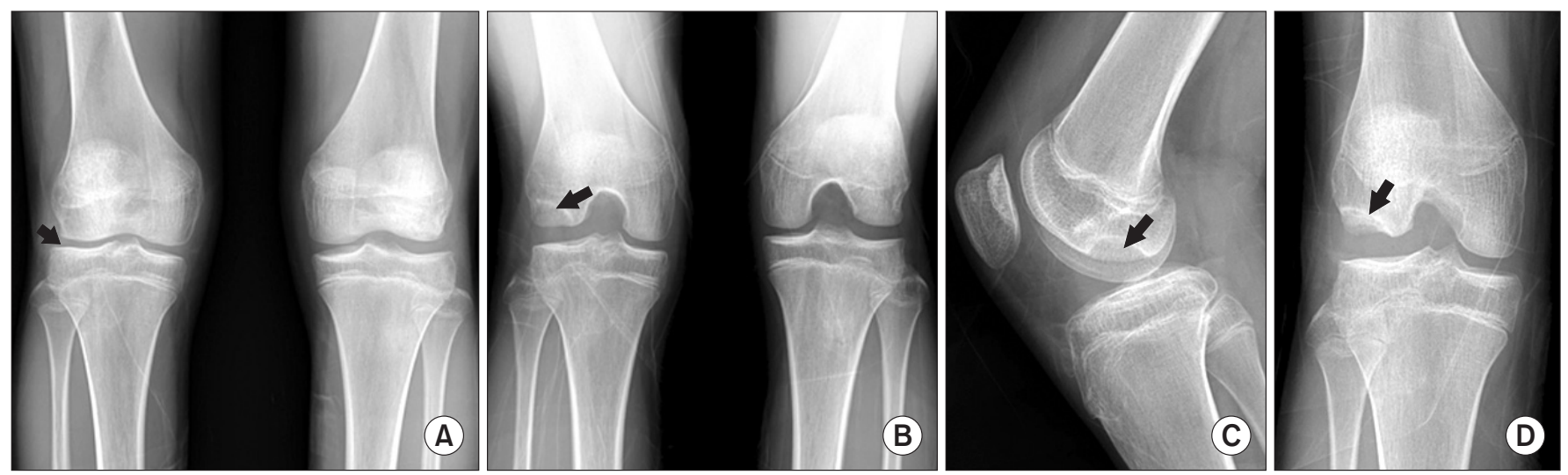

Fig. 1. (A) Standing anteroposterior view showing lateral joint space narrowing (arrow) of the right knee. (B) Rosenberg view showing the osteochondral lesion (arrow) in the posterolateral aspect of the right lateral femoral condyle. (C) Lateral view of the right knee showing the extent of the osteochondral lesion (arrow) in the posterior aspect of the lateral femoral condyle. (D) Tunnel view of the right knee showing the osteochondral lesion with defect (arrow) in the posterolateral aspect of the lateral femoral condyle. 

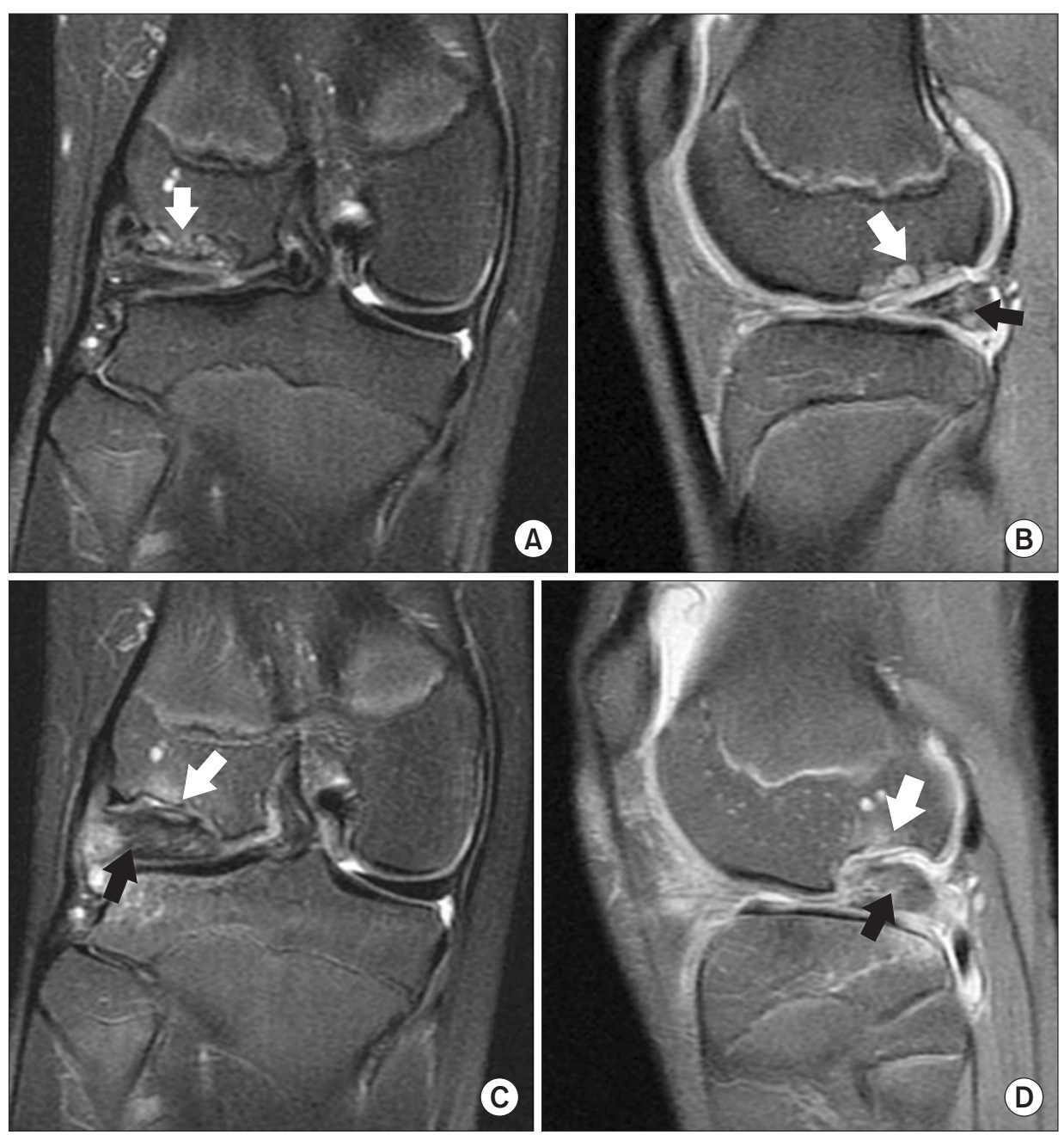

Fig. 3. Initial preoperative magnetic resonance imaging (MRI) performed at another hospital showing the osteochondral lesion with subchondral cysts (white arrow) in the posterior aspect of the right lateral femoral condyle (LFC) in coronal plane (A) and the osteochondral lesion (white arrow) with posteriorly displaced lateral discoid meniscus (black arrow) in the posterolateral aspect in sagittal plane (B). Follow-up (5 months after initial MRI) preoperative MRI performed at our hospital showing the more progressed osteochondral lesion (white arrow) in coronal plane (C) and the massive defect of the LFC (white arrow) with retorn lateral meniscus adhering to the LFC (black arrow) in sagittal plane (D).

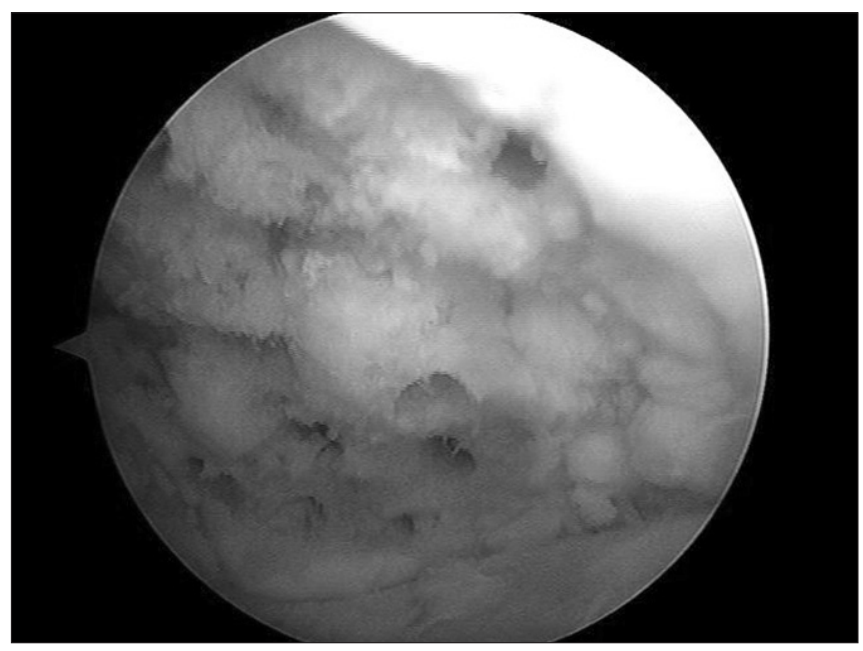

Fig. 4. The osteochondritis dissecans lesion treated with multiple drilling at another hospital showed the large osteochondral defect without any regeneration. plantation for deficiency of the lateral meniscus with joint space narrowing, considering the patient was a high-level gymnast and wanted early return to previous level of sports activities.

\section{Surgical Technique}

At one month after the first arthroscopic surgery performed at our hospital (5.5 months after the surgery performed at another hospital), she underwent osteoperiosteal autologous iliac crest graft implantation and lateral meniscus allograft transplantation as a one-stage procedure.

An anterolateral incision was used to expose the lateral compartment of the knee joint. The OCD lesion was identified and completely debrided, then multiple drilling using a fine 1.2-1.5$\mathrm{mm}$ diameter $\mathrm{K}$-wire was performed toward the lesion to break down the sclerotic avascular zone (Fig. 5). In the next step, the size and depth of the defect was measured as $1.6 \mathrm{~cm} \times 2.4 \mathrm{~cm}$ and $0.8 \mathrm{~cm}$, respectively using various-sized osteotomes. The anterior iliac crest was then accessed by making an incision $2-3 \mathrm{~cm}$ 
posterior to the anterior-superior iliac spine and an osteochondral fragment that matches the size of the lesion was harvested without damaging periosteum (Fig. 6). The defect in the iliac crest was backfilled with allograft bone from the lateral meniscus allograft. The harvested iliac crest plug with periosteum preservation was inserted into the lesion of the LFC and impacted by adjusting to the adjacent cartilage level (Fig. 6). The periosteum layer was placed slightly below the cartilage level. The following step for lateral meniscus allograft transplantation was performed using a "key-hole technique." But, the depth of the bone bridge was made to be $6 \mathrm{~mm}$ not to harm the growth plate of the tibia. Knee joint motion was performed to check the stability of the inserted iliac crest plug and the transplanted lateral meniscus allograft. Finally, the integrity and congruency of the grafts were

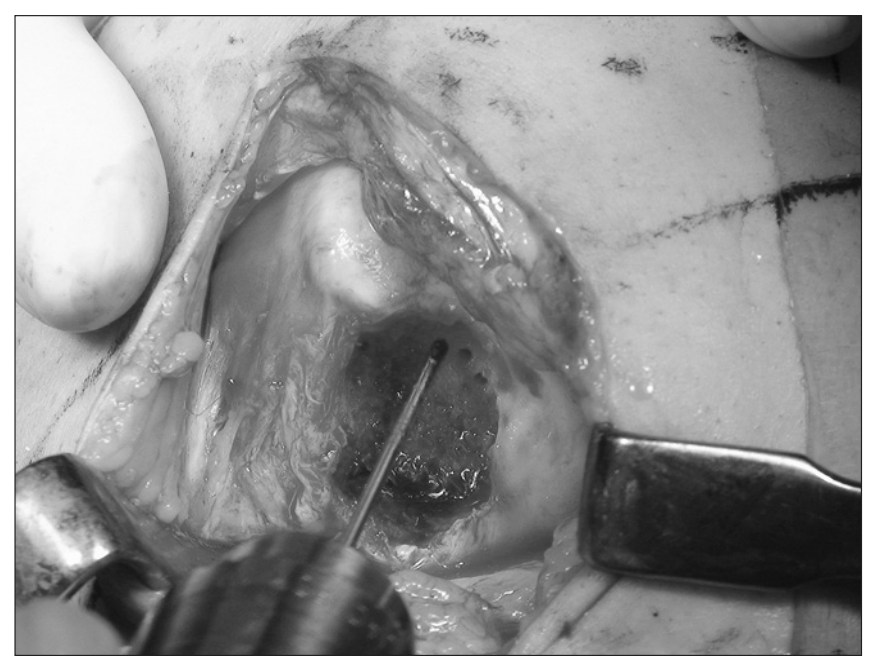

Fig. 5. Photograph of the exposed right lateral femoral condyle. The osteochondritis dissecans lesion was debrided and multiple drilling using $\mathrm{K}$-wire was performed toward the lesion. evaluated arthroscopically. After completion of the procedure, occurrence of bleeding from the healthy underlying bone resulting from multiple drilling was checked.

\section{Postoperative Rehabilitation}

The knee was immobilized in a cast during the first 3 weeks after surgery and protected using a brace for 12 weeks. Active quadriceps isometric exercise began on the first postoperative day. A $60^{\circ}$ range of motion (ROM) was allowed at 3 weeks and $90^{\circ}$ ROM at 6 weeks after surgery. Full weight bearing was allowed 6 weeks postoperatively. Light running was allowed at 3 months after surgery and return to sports at 6 months after surgery, although strenuous contact sports were prohibited.

\section{Follow-up Evaluation}

Plain radiography and 3-dimensional computed tomography (3D CT; Aquilion 64, Toshiba, Tokyo, Japan) were carried out on the first postoperative day, which showed a well integrated bone plug in the LFC and an allograft in the lateral tibial plateau (Fig. 7). At 24 months after surgery, the Lysholm score improved from 47 to 85, the International Knee Documentation Committee subjective knee form improved from 57 to 75, and the Tegner activity score improved from 4 to 8 . The visual analogue scale was 0 point. There was no donor-site complication such as pain and sensory loss as well as growth deformity. Isokinetic muscle strength test, follow-up MRI, and second-look arthroscopy were performed at 30 months after surgery. The deficit of isokinetic muscle strength (flexor peak torque/body weight) of the involved side was $9.2 \%$ and the hamstring-quadriceps ratio was 59\%. On follow-up MRI and second-look arthroscopy, the iliac crest plug was well incorporated with the LFC, and the OCD lesion healed with fibrous cartilage regeneration as well as stabilized lateral me-
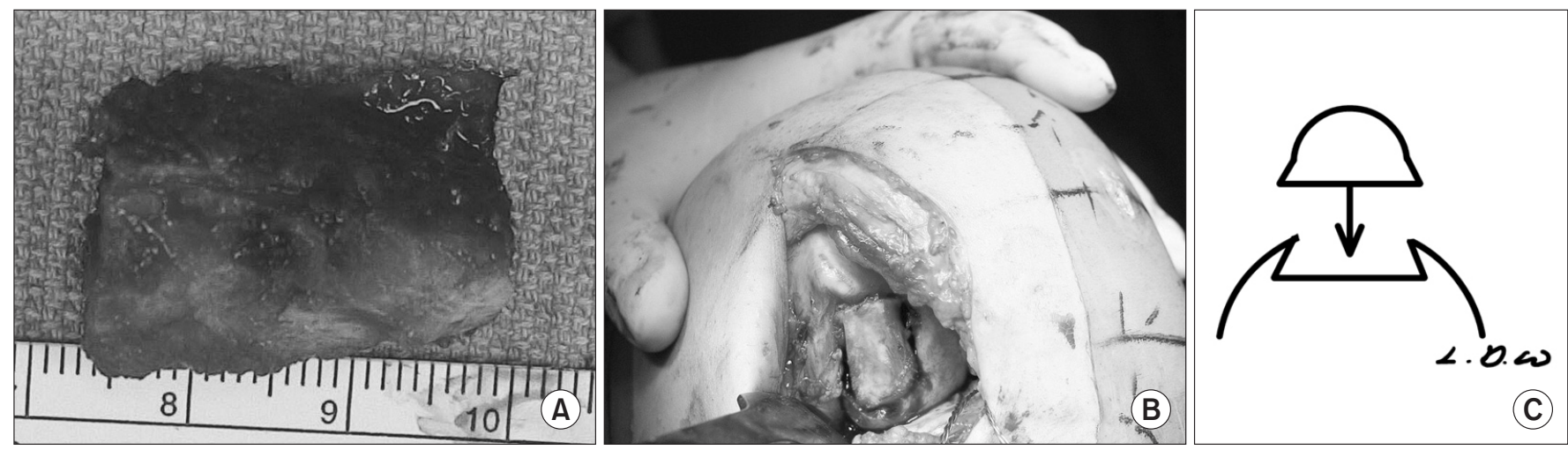

Fig. 6. Bone plug harvest and transplantation. (A) A bone plug matching the size of the osteochondritis dissecans lesion was harvested without damaging the periosteum. (B) The harvested iliac crest plug with periosteum preservation was inserted into the lesion of the lateral femoral condyle and impacted. (C) The press-fitting mortise wider and deeper at the articular surface was used during graft insertion. 


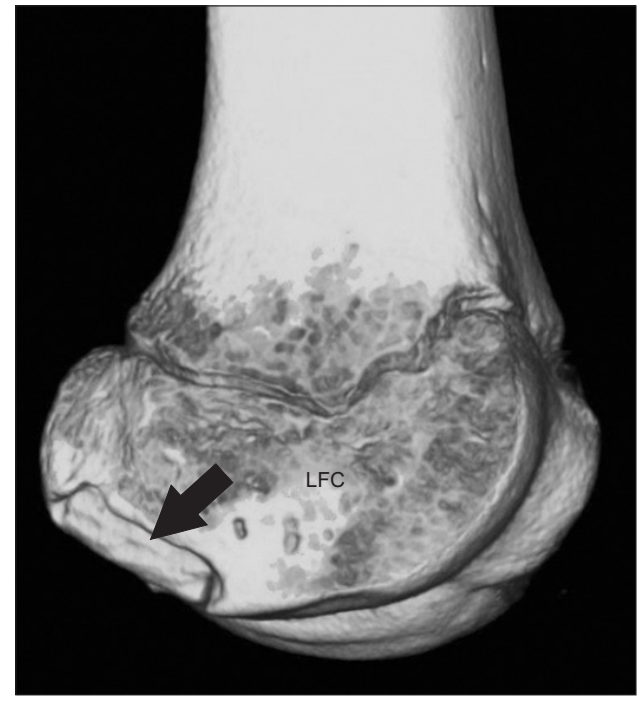

Fig. 7. Three-dimensional reconstructed computed tomography of the right knee revealing the stabilized periosteal plug (arrow) at the lateral femoral condyle (LFC). niscus allograft (Fig. 8). The surrounding cartilage revealed no progression of degeneration or formation of a kissing lesion. She could return to previous level of gymnastic activities without any swelling or pain at final evaluation at 24 months after surgery.

\section{Discussion}

This case report describes osteoperiosteal autologous transplantation for massive OCD combined with lateral meniscus allograft transplantation for deficiency of the lateral meniscus as a "onestep" technique. The patient could return to high-level sports activities as well as full daily activities without symptoms. In addition, a good morphological restoration with fibrous cartilage covering of the massive OCD lesion including osseous defect and well-maintained lateral meniscus allograft were shown on the follow-up MRI and second-look arthroscopy.

A discoid lateral meniscus sometimes results in OCD of the LFC, which has been reported in co-presence with discoid meniscus in up to $19 \%^{1)}$. The discoid meniscus, regardless of tear, could be an important risk factor for OCD, because it might
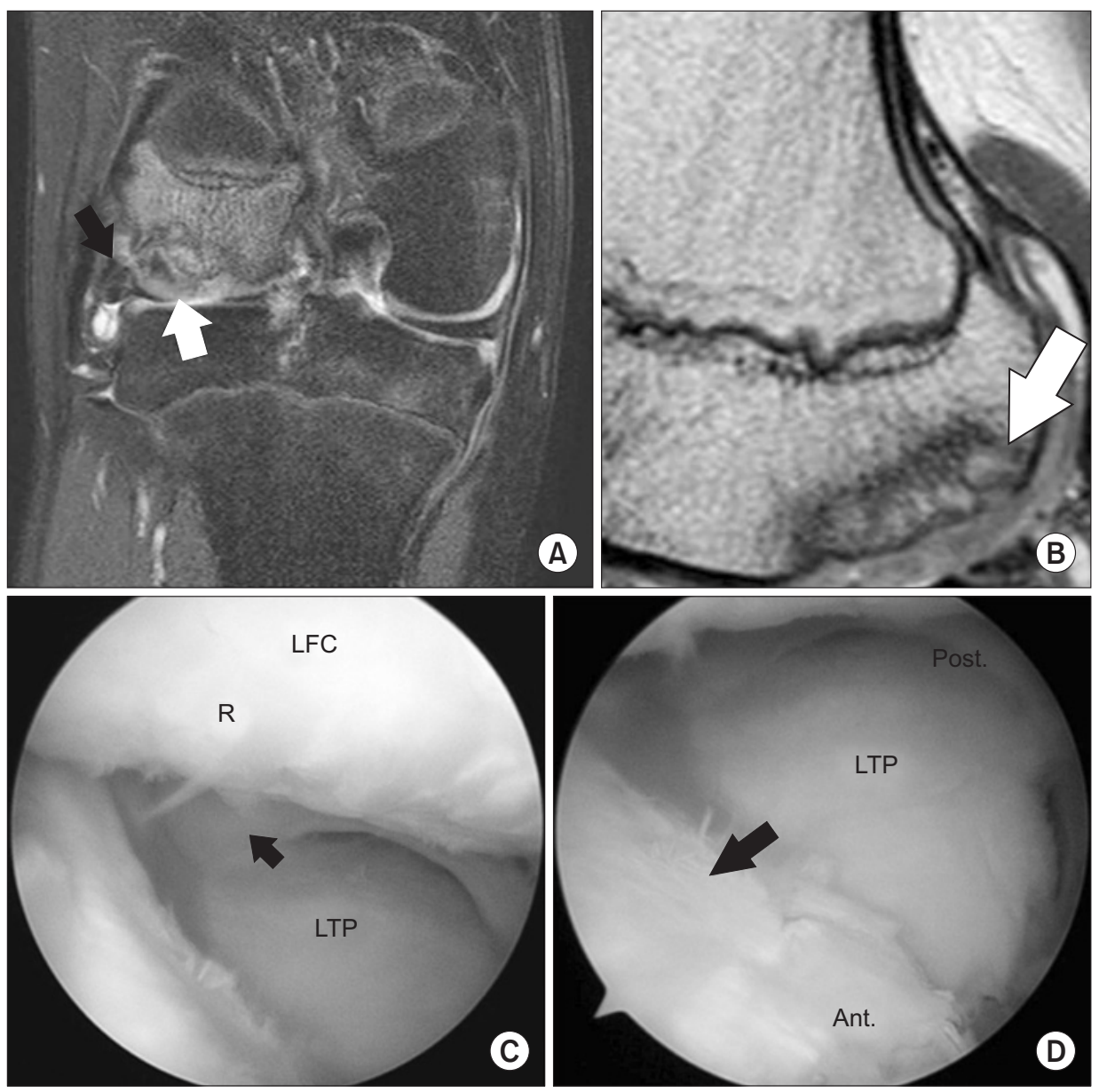

Fig. 8. Follow-up magnetic resonance imaging showed well incorporated periosteal plug (white arrow) and lateral meniscus allograft (black arrow) in the posterior aspect of the right LFC in coronal plane (A) and well integrated periosteal plug with surrounding cartilage of the LFC (white arrow) in sagittal plane (B). Second-look arthroscopy showed well covering (R) previous osteochondritis disseccans lesion without degeneration $(\mathrm{C})$ and well maintained lateral meniscus allograft without displacement (black arrow) (C, D). LFC: lateral femoral condyle, $\mathrm{R}$ : regenerated cartilage, LTP: lateral tibial plateau, Post: posterior, Ant: anterior. 
produce repetitive abnormal stress on the immature osteochondral bone in patients who are still growing and participate in sports activities ${ }^{1)}$. These phenomena would be more accelerated after total resection of the discoid meniscus as in our patient $t^{2}$. Hence, we performed lateral meniscus allograft transplantation concomitantly, despite the patient's young age. Although there has been paucity of literatures on the outcomes of meniscus transplantation in children or juveniles and it has not yet been considered as an optimal treatment for the meniscus-deficient knee in patients with immature skeleton, meniscus preservation or restoration has been suggested as an alternative treatment for young patients with use of improved techniques of meniscus allograft transplantation ${ }^{3}$. Andrish ${ }^{3)}$ noted that the best candidate for meniscus transplantation could be young patients who had minimal degenerative joint disease. We could perform lateral meniscus transplantation with a bone block technique without any violation of the physis of the tibia by making a shallow bone hole after failure of meniscectomy with repair at another hospital.

Several techniques such as multiple drilling, osteochondral autograft transplantation, and osteochondral allograft transplantation for OCD of the knees have been evolved. Osteochondral autograft transplantation can be used to treat osteochondral lesions with a large defect providing stability between cartilage and osseous levels ${ }^{2}$. However, obtaining large plugs in the osteochondral autograft transplantation, so called mosaicplasty, could result in significant donor-site morbidity ${ }^{4)}$. For this reason, there have been several attempts to obtain autologus osteochondral grafts from other sites. Espregueira-Mendes et al. ${ }^{5)}$ developed autologous osteochondral grafting from the upper tibio-fibular joint for the treatment of knee cartilage lesions. Even though the upper tibiofibular joint does not play a significant role, there are still problems of donor site morbidity such as disruption of the proximal joint or risk of peroneal nerve injury through the lateral approach. This technique was not suitable for high-level gymnast. Leumann et al. ${ }^{2)}$ designed a modified mosaicplasty for severe OCD of the talus using bony periosteum-covered iliac crest plug transplantation and showed good radiological results in $82 \%$ at a mean follow-up of 25 months. The anterior part of the iliac crest has been widely used for autologous bone grafting including cancellous bone and structured bone ${ }^{2}$. In addition, the periosteum of membrane bone was identified to have abundant stem cells with the chondrogenic capacity and to provide mechanical stability and biological interaction ${ }^{6}$. Matsushima et al. ${ }^{6}$ reported that the ileum represented relatively high gene expression of type I collagen and type II collagen compared with cranial or mandibular bone. O'Driscoll and Fitzsimmons ${ }^{7}$ demonstrated that the chondrocyte precursor cells exist in the cambium layer of periosteum and they could meet the requirements as a source of cells, a scaffold, and a source of growth factors and revealed that dynamic fluid pressure increases cartilage formation. Most of these articles were laboratory studies, not clinical studies, except for the study of Leumann et al. ${ }^{2}$.

Peterson et al. ${ }^{8)}$ reported that $91 \%$ of 58 patients with advanced OCD lesions treated with autologous chondrocyte transplantation showed good or excellent clinical results after a mean followup of 5.6 years. In 7 of 58 patients with a deep defect over $8 \mathrm{~mm}$, the defect was filled with cancellous bone after removal of the subchondral bone, and autologous cultured chondrocyte transplantation was performed using a double-layered "sandwich technique". This technique could be considered as a treatment option in our current patient who had a large bony defect with cartilage loss. However, autologous chondrocyte implantation requires two or three operative procedures and it takes long time for one stage operation with lateral meniscus allograft transplantation. Hence, we determined to perform "one-step" osteoperiosteal autologous iliac crest graft and lateral meniscus allograft transplantation.

Contrary to the osteochondral autograft transplantation, the periosteum reproduces fibrous cartilage, ${ }^{2,7}$. Although hyaline cartilage has been known to be superior to fibrous cartilage in terms of biomechanical property and longevity, there are some reports showing the regenerated fibrous cartilage is comparable to the grafted hyaline cartilage. Knutsen et al..$^{9)}$ performed a comparison study of clinical outcomes between the fibrous cartilage and the hyaline cartilage after microfracture and ACI, respectively and concluded that there were no significant differences in clinical and radiological results in the mid-term follow-up. Gobbi et al. ${ }^{10)}$ also showed no significant differences in terms of clinical results between them in the ankle joint.

It is critical to provide a good congruency of articular cartilage in osteochondral autograft transplantation avoiding step off, which strongly related to better clinical outcomes in the longterm follow-up. In our patient, we used press-fitting mortise, which is wider and deeper at the articular surface, assuming that it could have stronger pullout strength (Fig. 6).

Although the short-term follow-up and lack of cases were the most significant limitations of this report, the modified osteochondral autograft transplantation demonstrated several advantages such as reproducibility, lower risk of donor site morbidity, and provision of fibrous cartilage coverage as well as filling of the bone defect.

In conclusion, this osteoperiosteal autologous iliac crest graft transplantation could be a strategy for a joint salvage procedure 
with restoration of the osteochondral defect, reproduction of the fibrous cartilage, and mechanical stability as well as biological healing for massive OCD lesions. Additional aggressive treatments for combined torn discoid lateral meniscus, especially for deficiency of the meniscus, should be considered to prevent failure of the treatment of OCD. The successful results of our "onestep" technique should be validated in further studies with longterm evaluation.

\section{Conflict of Interest}

No potential conflict of interest relevant to this article was reported.

\section{References}

1. Deie M, Ochi M, Sumen Y, Kawasaki K, Adachi N, Yasunaga Y, Ishida O. Relationship between osteochondritis dissecans of the lateral femoral condyle and lateral menisci types. J Pediatr Orthop. 2006;26:79-82.

2. Leumann A, Valderrabano V, Wiewiorski M, Barg A, Hintermann B, Pagenstert G. Bony periosteum-covered iliac crest plug transplantation for severe osteochondral lesions of the talus: a modified mosaicplasty procedure. Knee Surg Sports Traumatol Arthrosc. 2014;22:1304-10.

3. Andrish JT. Meniscal injuries in children and adolescents: diagnosis and management. J Am Acad Orthop Surg. 1996; 4:231-7.

4. Valderrabano V, Leumann A, Rasch H, Egelhof T, Hinter- mann B, Pagenstert G. Knee-to-ankle mosaicplasty for the treatment of osteochondral lesions of the ankle joint. Am J Sports Med. 2009;37 Suppl 1:105S-111S.

5. Espregueira-Mendes J, Pereira H, Sevivas N, Varanda P, da Silva MV, Monteiro A, Oliveira JM, Reis RL. Osteochondral transplantation using autografts from the upper tibio-fibular joint for the treatment of knee cartilage lesions. Knee Surg Sports Traumatol Arthrosc. 2012;20:1136-42.

6. Matsushima S, Isogai N, Jacquet R, Lowder E, Tokui T, Landis WJ. The nature and role of periosteum in bone and cartilage regeneration. Cells Tissues Organs. 2011;194:320-5.

7. O'Driscoll SW, Fitzsimmons JS. The role of periosteum in cartilage repair. Clin Orthop Relat Res. 2001;(391 Suppl): S190-207.

8. Peterson L, Minas T, Brittberg M, Nilsson A, Sjogren-Jansson E, Lindahl A. Two- to 9-year outcome after autologous chondrocyte transplantation of the knee. Clin Orthop Relat Res. 2000;(374):212-34.

9. Knutsen G, Drogset JO, Engebretsen L, Grontvedt T, Isaksen V, Ludvigsen TC, Roberts S, Solheim E, Strand T, Johansen O. A randomized trial comparing autologous chondrocyte implantation with microfracture: findings at five years. J Bone Joint Surg Am. 2007;89:2105-12.

10. Gobbi A, Francisco RA, Lubowitz JH, Allegra F, Canata G. Osteochondral lesions of the talus: randomized controlled trial comparing chondroplasty, microfracture, and osteochondral autograft transplantation. Arthroscopy. 2006;22: 1085-92. 